\title{
UNA MIRADA CRÍTICA AL SECTOR \\ EMPRESARIAL COREANO, CON ESPECIAL REFERENCIA A ARGENTINA
}

\section{A critical view of the Korean business sector, with special reference to Argentina}

\author{
M. Pilar Alvarez * y Luciana Manfredi ${ }^{* *}$
}

\section{Resumen}

El presente trabajo se propone analizar las relaciones comerciales entre Corea del Sur y Argentina mediante un estudio comparado sobre las empresas medianas y multinacionales coreanas que deciden invertir en el país. Se hará hincapié en las variables sociales, culturales y políticas que limitan o alientan el afianzamiento de lazos comerciales con el fin de poder comprender los lineamientos de cambio necesarios, desde una mirada coreana, para consolidar una relación sostenible en el largo plazo.

Palabras clave: relaciones comerciales, Corea del Sur, Argentina, empresas multinacionales, empresas medianas.

" Investigadora, CONICET, Buenos Aires.<mpam1979@gmail.com>

** Profesora asistente, Universidad Icesi, Cali, Colombia. lcmanfredi@icesi.edu.co Recibido el 20 de julio de 2012; aceptado el 5 de diciembre de 2012. 


\section{Abstract}

The goal of the present research is to analyze trade relations between South Korea and Argentina by comparing Korean medium-sized firms and multinationals which decide to invest in our country. We will pay special attention to social, cultural and political variables that limit or encourage the reinforcement of trade bonds aimed at better understanding changes required, from a Korean perspective, to consolidate sustainable relation in the long term.

KeYwords: trade relations, South Korea, Argentina, multinationals, medium-sized firms 


\section{INTRODUCCIÓN}

Las relaciones bilaterales entre Corea del Sur ${ }^{1}$ y Argentina se remontan al decenio de los años sesenta. Signadas por la distancia geográfica, la ignorancia mutua y la falta de estrategia por parte del gobierno argentino, ellas adquirieron un ritmo irregular y asimétrico. En este sentido, la disyuntiva en que se inscribe la cooperación entre ambos países plantea una serie de interrogantes relacionados con los factores que han producido, por un lado, el reciente éxito empresarial de algunas multinacionales coreanas que operan en el país y, por otro, el crítico rol de las pequeñas y medianas empresas coreanas que intentan insertarse en el mercado local.

Un factor decisivo en este escenario ha sido el rol de la comunidad coreana en el país. La presencia de más de 40 mil coreanos en Argentina antes de la crisis del 2001 permitió desarrollar un lazo cultural mucho más sólido que en otros países de América Latina. Si bien en la actualidad el número de residentes ha descendido a unos 25 mil, el otro ha dejado de ser un mero desconocido para convertirse en parte integral de la cultura local. A este fenómeno se suma la llegada reciente del Hally ${ }^{2}$ que a través de la música, el cine y las telenovelas ha logrado una expansión

De aquí en adelante Corea

Significa «Ola Coreana» y se refiere a la exitosa expansión de los productos culturales coreanos en el exterior. de lo «coreano» en diversos sectores sociales de la población local.

Sin embargo, a pesar de que la distancia geográfica se diluye en un proceso acelerado de acercamiento sociocultural, las relaciones comerciales parecieran no acompañar el ritmo de dicha integración. ¿Cuáles son las principales dificultades que encuentran los inversores coreanos? ¿Cuáles son los agentes económicos más afectados: medianas empresas (importadoras, exportadoras), multinacionales? ¿Las limitaciones para la inversión coreana en el país radican en discrepancias socioculturales, en ambivalencias de la política comercial local o en una combinación de ambas? Con el fin de poder responder a estos interrogantes, el presente artículo analizará en forma comparada el rol de las medianas empresas en el país con las nuevas estrategias de las multinacionales LG y Samsung.

Primero se reseñará brevemente la historia de las relaciones CoreaArgentina y a continuación se analizarán críticamente los avances, límites y desafíos de las firmas coreanas en el país. Debido a la falta de trabajos previos sobre esta temática decidimos crear un corpus conformado por datos estadísticos sobre los flujos comerciales y las principales importadoras y exportadoras coreanas en el país suministrados por la Korean International Trade Association (KITA), entrevistas a miembros de la Korea Trade-Investment Promotion Agency (KOTRA), empresarios coreanos en Argentina y expertos 
en integración regional Corea-América Latina. A partir del estudio de la información obtenida nos atrevemos a afirmar que quienes encuentran mayores dificultades son las empresas medianas debido a que la política comercial local no ofrece garantías reales a sus posibles inversiones. Sin embargo, para las multinacionales las ambivalencias políticas y económicas no parece ser un condicionamiento importante, en los últimos años -en el marco política nacional orientada al consumo- han logrado una inserción cada vez más exitosa.

\section{LA INTERNACIONALIZACIÓN EMPRESARIAL: UNA}

\section{MIRADA CRÍTICA}

En el marco de la globalización, la internacionalización de las empresas ha sido clave no solo para su expansión y crecimiento sino también para la consolidación de las economías locales en el mundo.

Si bien el actual escenario internacional provee a las empresas mayor acceso a mercados extranjeros y diferentes recursos, la internacionalización extranjera -definida como el proceso por el cual las firmas se mueven hacia fuera del país de origen (Barkema y Bell, 1996)- tiene varios riesgos y desafíos. Cada vez que una compañía decide penetrar en un nuevo mercado, debe afrontar ciertos niveles de incertidumbre y riesgo propios de aventurarse a cruzar la frontera nacional.
La literatura sobre el tema plantea que existen varios factores que es importante tener en cuenta cuando una empresa decide internacionalizarse (Root, 1998). Estos factores pueden ser tanto externos como internos. Entre los más destacados encontramos: el mercado y la demanda potencial, la cultura nacional extranjera, la producción, la posibilidad de crecimiento y de generación de economías de escala, la distancia geográfica y el ambiente. Asimismo, existen otras variables relacionadas con la categoría de factores ambientales y con los elementos económicos, socioculturales, políticos e institucionales, que son relevantes al momento de elegir el país de destino en el proceso de internacionalización. Entre estos cabe destacar el sistema político, el sistema de partidos políticos, el grado de institucionalización de la democracia (que incluye el respeto por los derechos fundamentales y los derechos laborales), las instituciones y reglas de juego, el respeto por la propiedad privada y la estabilidad política e institucional del país de destino.

De los aspectos mencionados, al momento de invertir en el extranjero suelen tener un peso decisivo las políticas comerciales, así como las regulaciones y normas que afectan los negocios internacionales. Mientras que las compañías buscan marcos políticoeconómicos adecuados para conciliar sus intereses productivos, como bien sostiene Alicia Bárcena de la Comisión Económica para América Latina y el Caribe (CEPAL), los Estados buscan 
inversión extranjera que contribuya «a desarrollar sectores estratégicos, transformar la estructura productiva, acumular conocimiento y contribuir al incremento y la calidad del empleo». ${ }^{3}$ En este sentido, la Argentina presenta tensiones negativas (desde la perspectiva de la empresa usualmente centrada en la rentabilidad) y positivas (desde la mirada oficial de reforzar la industrialización local). Como el presente trabajo se centra en las dificultades y logros de las empresas coreanas en el país, este segundo aspecto no será analizado desde el punto de vista de su impacto en la economía argentina sino desde el de la inversión coreana.

De acuerdo con las cifras preliminares de la Unctad para 2011, ese año la región de América Latina y el Caribe recibió inversiones por un monto total de 216.400 millones de dólares, es decir, $34,6 \%$ más que durante 2010, lo que la posicionó como la región de mayor crecimiento, seguida por Europa del Este. En cambio, ese año las economías desarrolladas en su conjunto recibieron $18,5 \%$ más IED que en 2010. Entre 2010 y 2011 las principales economías latinoamericanas registraron un incremento de los flujos de IED, salvo la Argentina y México, donde esta tuvo una caída de $8,8 \%$. Adicionalmente, según los datos de la Unctad, en 2011 la IED que ingresó en la Argentina sumó 6300 millones de dólares, mientras que las corrientes de inversión recibidas

3 www.lanacion.com.ar Domingo 16 de diciembre de 2012 "Capital que queda prisionero en el país» por economías más pequeñas, como Colombia o Chile, fueron de 14.400 y 17.600 millones, respectivamente. ${ }^{4}$

De acuerdo con lo que plantea la teoría sobre la internacionalización previamente aludida, el marco institucional político-económico argentino presenta diversas complejidades que contradicen y limitan la inversión extranjera. Siguiendo esta línea de estudio, la Teoría del Costo Transaccional, paradigma dominante para explicar la decisión del modo de entrada a mercados extranjeros (Lin, 2000), una empresa selecciona el modo de ingresar a un país extranjero considerando la forma más eficiente de gobernanza. Parmigiani (2007) sostiene que los factores ambientales son los que representan mayores desafíos para una empresa que intenta internacionalizarse dado que están directamente relacionados con los altos niveles de incertidumbre producidos por un potencial cambio en el ambiente.

Frente a este escenario, ¿cuáles son los principales obstáculos que deben sortear las empresas coreanas? ¿Cómo han impactado los recientes cambios en la estrategia comercial argentina? Antes de responder a estos interrogantes (en la sección 3), consideramos fundamental historiar brevemente las relaciones Corea-Argentina que le han dado forma a las actuales dinámicas comerciales entre ambos países.

4 www.lanacion.com.ar Miércoles 25 de enero de 2012 «En 2011 cayó la inversión externa que llegó al país» por José Hidalgo Pallares. 
2. LAS RELACIONES

Bilaterales CoREA DEL

Sur-Argentina

Los años sesenta marcan el comienzo de las relaciones oficiales entre Corea y Argentina. En febrero de 1962, culminadas las conversaciones entre representantes de ambos países en Buenos Aires, Washington y Tokio, el Embajador Extraordinario y Plenipotenciario de Argentina en Japón, Alejandro Orfila, y el representante del gobierno coreano, Embajador Pai Ei Whan, fueron los encargados de establecer relaciones diplomáticas. El primer embajador coreano en la Argentina fue Chung Il Kwon, Embajador en los Estados Unidos y concurrente en nuestro país; la embajada se abrió en diciembre de 1963 con Bae Ui Hwan como primer embajador en Buenos Aires, quien finalizó su mandato en 1967. Por su parte, la Argentina abrió su embajada en Seúl en marzo de 1966 a cargo del Encargado de Negocios y Ministro Plenipotenciario Alejandro Galarce, apertura enmarcada en el fortalecimiento de los lazos entre ambos países dentro de las llamadas «fronteras ideológicas» ${ }^{5}$. Sin embargo, dos años más tarde la Embajada se cerró, quedando desde el 6 de octubre de 1969 el Embajador Argentino en Japón como concurrente en Corea.

Mera, Carolina (2007), «Panorama general y reflexiones acerca de las relaciones entre Argentina y Corea» en Revista de Estudios Internacionales, $\mathrm{N}^{\circ}$..., p. 69.
A pesar de ciertas irregularidades iniciales ${ }^{6}$, en los años setenta cuestiones ideológicas -afinidad anticomunista entre Park Chung Hee y Videla-, culturales $^{7}$, sociales -migración ${ }^{8}-$ y comerciales dinamizaron la incipiente relación existente entre ambos países.

Durante la llamada «década perdida» en América Latina, las relaciones comerciales entre Corea y Argentina no experimentó grandes avances (véase el Apéndice 4). Sin embargo, por aquellos años cobró fuerza el flujo migratorio en la región. Como señala Mera (2010), el máximo exponente se alcanzó en los años noventa cuando se registraron 42 mil coreanos residentes en Argentina, la mitad de los cuales habrían llega-

6 El primer embajador coreano en Argentina finalizará su misión en 1967, nombrándose al siguiente embajador, Kim Dong Sung, recién en marzo de 1976. La embajada argentina en Seúl cerró sus puertas el 6 de octubre de 1969 otorgándole la jurisdicción al Embajador argentino en Japón. Por otra parte, existe sólo un acuerdo establecido en estos años: "Convenio Cultural entre la República de Corea y la República Argentina» firmado el 8 de agosto de 1968 en Buenos Aires (Ley 18.387) que entró en vigor el 4 de enero de 1970.

Firma del «Acta de la Comisión Mixta Cultural Argentina-Coreana Sección Buenos Aires» el 28/11/75 y el «Acta de la I Reunión de la Comisión Mixta Cultural Argentina-Coreana Sección Buenos Aires» el $5 / 10 / 75$.

8 Recién se puede hablar de una inmigración coreana en la Argentina a partir de 1965. A fines de los años 70, como parte de un programa del gobierno surcoreano de emigración en grupo, llegaron al país unas 500 familias. 
do entre 1984 y 1989, firmándose en 1985 el $\nabla$ Acta de Procedimiento para el Ingreso de Inmigrantes Coreanos a la Argentina». De acuerdo con datos del Ministerio de Relaciones Exteriores de Corea del Sur, se estima que en 2010 vivían en el país unos 25 mil coreanos, una constante desde 2001, cuando el número de residentes ya había descendido notablemente.

El flujo migratorio transformó el eje de las relaciones entre ambos países, convirtiéndose Argentina en la fuente más importante de lazos culturales de la región, como lo revelan la organización del Primer Encuentro de Estudios Coreanos de América Latina (2003), la creación del Centro Corea-Argentina (2005) - áreas de estudios coreanos más pequeñas en universidades nacionales ubicadas en La Plata, Córdoba, Rosario, Tucumán y Comahue -, la apertura del Centro Cultural Coreano en América Latina con sede única en Buenos Aires (2006), la organización del concurso K-Pop y el aumento del número de becarios.

A nivel político, los principales avances lo constituyen, por un lado, la visita al país en 1996 del Presidente Kim Young Sam, durante la cual se firmó una serie de acuerdos que buscaban promover las relaciones institucionales de cooperación económica y por el otro, el otorgamiento (2004) por el ex presidente Roh Moo Hyun del primer crédito tras la declaración de cesación de pagos de la deuda externa en diciembre de 2001. En 2006 se suscribió el Acuerdo sobre Cooperación Económica y Comercial entre ambos gobiernos que abarca temas tales como el desarrollo de bioenergía, medidas sanitarias y fitosanitarias de producción agrícola, cuestiones aduaneras, etc. Y en 2007, se creó el Centro para la Cooperación Coreano-Sudamericana en Energía y Recursos Naturales.

A nivel comercial, el intercambio aumentó significativamente en las últimas décadas (véase el Apéndice 2) pasando de 90 millones de dólares a 360 millones de dólares y a 330 millones (2006) por concepto de exportaciones coreanas, 556 millones de dólares en importaciones y 50 proyectos de inversión por parte de Corea de 390 millones en 2006 y 910 millones en exportaciones, 800 millones en importaciones y 160 millones en inversión en 2010. En 2007, la Argentina exportó a Corea por valor de 636 millones de dólares e importó por 412 millones de dólares. El 95\% del total exportado se concentró en 10 productos: minerales de cobre, harina, aceite de soja, maíz, cueros y pieles curtidos, pescados congelados, quesos mozzarella, tubos de hierro o acero sin soldadura, vinos y mostos de uva y aceite de girasol. Por el contrario, entre los principales productos importados de Corea se destacan los bienes intensivos en tecnología, como partes para aparatos eléctricos y electrónicos (televisores y radios), vehículos, máquinas, hornos microondas y lavarropas, teléfonos celulares, plásticos e insumos agrícolas. 
Cuadro 1: Comercio Corea-Argentina 2002-2010

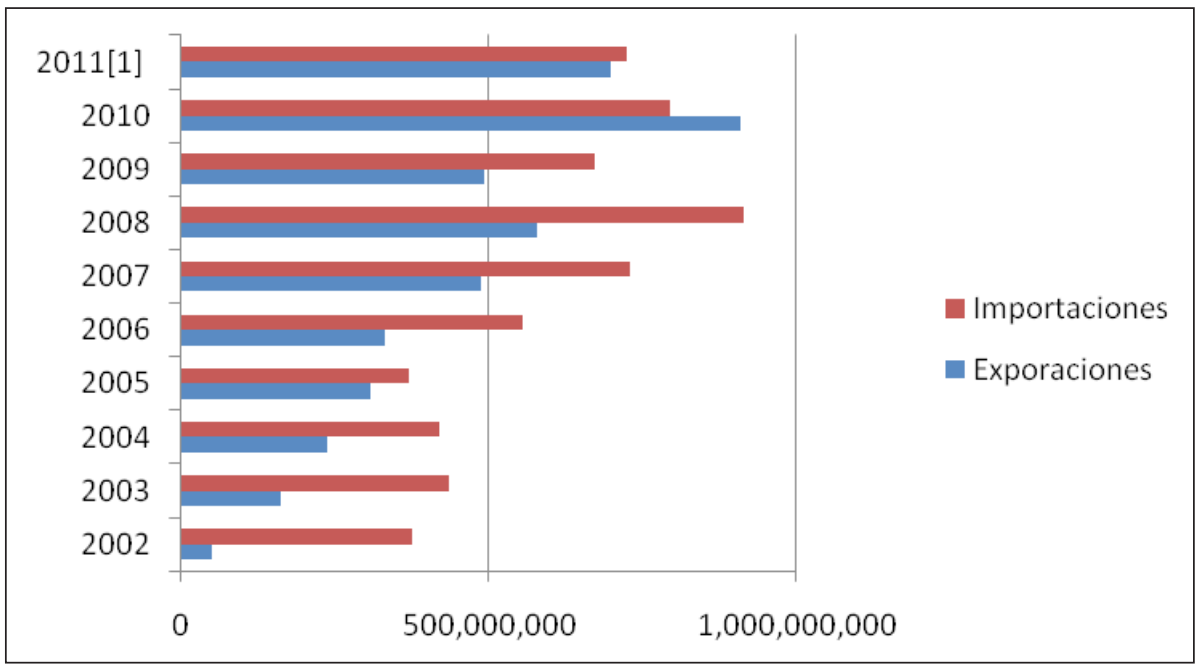

Fuente: Korean International Trade Association (KITA)

Respecto de las relaciones con MERCOSUR también observamos una dinamización de las relaciones comerciales, que pasaron de 1.354.715 dólares por concepto de exportaciones y 1.630.600 de importaciones en 2002 a 9.878.849 dólares y 4.829.499 dólares respectivamente en exportaciones e importaciones en 2011 (datos hasta el mes de agosto). En los últimos dos años la disparidad entre exportaciones e importaciones es cada vez más pronunciada (Cuadro 2). Asimismo, en términos regionales (véase el Apéndice 1) en 2010 Argentina ocupó el quinto lugar en las importaciones de América Latina, con un monto de 79.606.929 dólares, por debajo de Brasil, Chile y México; y el noveno lugar en el de exportaciones, con un monto de 909.449.459 dólares, muy por debajo de Brasil, México y Panamá.
Uno de los cambios más sorprendentes es la familiaridad de las marcas coreanas en el país. Ha pasado a ser común ver circular vehículos Hyundai, Daewoo, Kia Motors o electrodomésticos y electrónicos LG y Samsung. Dicho auge se ha beneficiado por la recuperación económica argentina -postcrisis 2001- y las nuevas estrategias de marketing promovidas por Samsung y LG han alcanzado un alto nivel de inserción en el mercado local de celulares, pantallas LCD y monitores. En este escenario cabe preguntarse cuáles son los obstáculos y alcances que definen las estrategias comerciales de estas multinacionales en Argentina y cuál es el rol de los medianos inversores coreanos. 
M. del Pilar Alvarez y Luciana Manfredi • Una mirada crítica al sector empresarial...

Cuadro 2: Comercio Corea-MERCOSUR

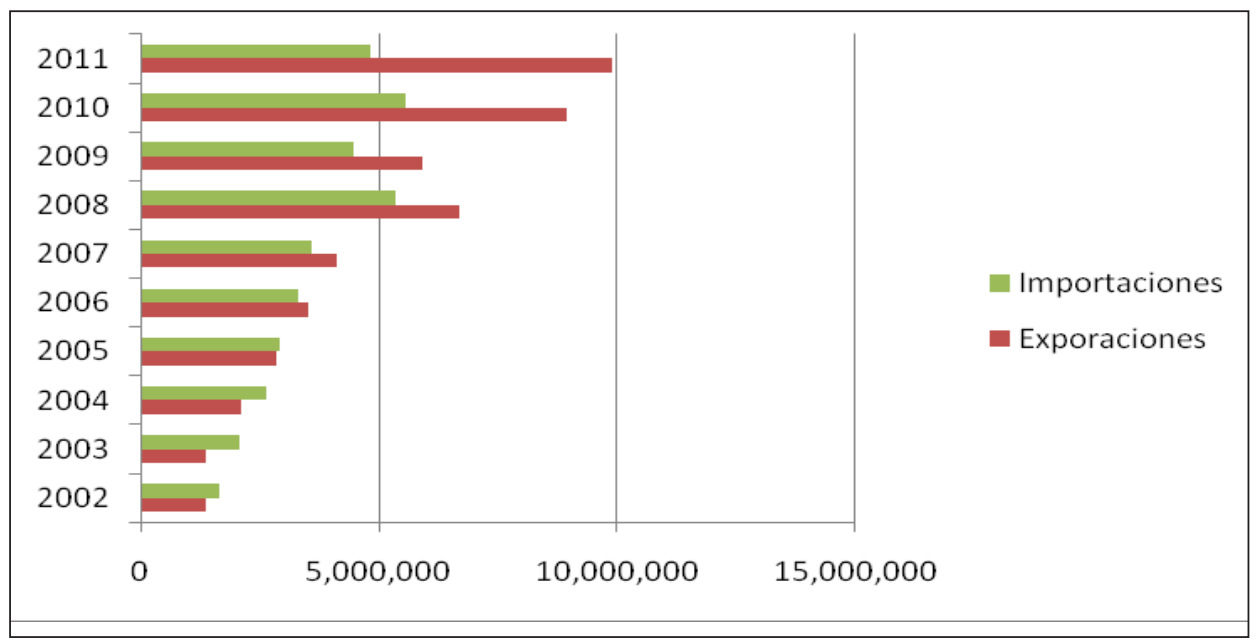

Fuente: Korean International Trade Association (KITA)

3. Oportunidades, AlCANCES Y LIMITACIONES

\subsection{Inversión, estrategias y multinacionales}

La política comercial exterior coreana se caracterizó en un comienzo por una intención política clara de estimular la diversificación regional de las exportaciones mediante la penetración en mercados emergentes y luego, hacia fines de los años noventa frente a las exigencias de la globalización - entendida como estandarización global, modernización y renovación-y falta de competitividad, las empresas implementaron una nueva estrategia de inversión directa en el extranjero para sustituir la producción interna, favoreciendo así, aunque en menor medida, el impulso comercial con América Latina. ${ }^{9}$

El ex presidente Roh Moo Hyun (2003-2008) y en especial el presidente Lee Myung Bak (2008-2013) ${ }^{10}$ se han orientado hacia la firma de tratados de libre comercio (TLC) con países y regiones clave y América Latina no ha sido excepción. En la actualidad, Corea posee TLC con Chile (el primero de la región), Singapur, EFTA, ASEAN, India, UE, Perú y Estados Unidos. Se han celebrado tratados con Colombia y Turquía, se encuentran en negociación

$9 \quad$ Para más detalles ver: Won Ho Kim (2000), La globalización de la economía coreana y América Latina. Disponible en: http:// www.asiayargentina.com/cari-12.htm

10 La nueva presidenta Park Geun Hye, electa el pasado diciembre no ha hecho ninguna mención de las políticas de comercio exterior que se implementarán con Argentina. 
con Canadá, México, GCC, Australia, Nueva Zelandia, China, Vietnam, Indonesia, China-Japón, y la Regional Comprehensive Economic Partnership (RCEP) estudió la posibilidad de celebrarlos con Japón, Israel, Centroamérica, Malasia y el Mercosur. ${ }^{11}$ Asimismo, existen acuerdos de cooperación agrícola recientemente con Paraguay y de intercambio tecnológico para el desarrollo del litio con Bolivia. Se han afianzado las relaciones con socios tradicionales de la región como Brasil y Panamá, y se han instalado empresas coreanas en Guatemala, El Salvador, República Dominicana, Honduras, etc.

En este contexto, las relaciones empresariales entre la Argentina y Corea se han profundizado gracias a una política planificada del Estado coreano y de las empresas multinacionales en la región. Esta relación asimétrica se ha favorecido por la política orientada al consumo y el crecimiento económico de los gobiernos Kirchner. De acuerdo con las estadísticas proporcionadas por KOTRA, en 2009 entre mil y 1200 empresas mantenían un flujo comercial con el país, pero solo Samsung Electronics, LG Electronics, Ceragem, Daewoo, Nugabest y CJ. LG y Samsung han instalado sus fábricas mediante convenios con fabricantes locales, si bien se dedican a ensamblar bienes con partes importadas o a distribuir productos terminados en el país.

11 Datos del Ministerio de Relaciones Exteriores de Corea del Sur (enero 2013): http://www.mofat.go.kr/ENG/policy/fta/ status/overview/index.jsp
Los rubros de las empresas coreanas que mantienen relaciones comerciales con Argentina incluyen una amplia gama que comprende productos químicos, láminas, tintas, autopartes, y construcción, entre otros. El monto de la inversión se calcula entre 20 y 50 mil millones de dólares, siendo el sector de mayor interés las energías renovables. De acuerdo con datos publicados por la Korean International Trade Association (KITA), la mayoría de las exportadoras se concentran en productos manufacturados como maquinaria y productos electrónicos, mientras que las importadoras lo hacen en productos alimenticios.

En una entrevista realizada en 2010, funcionarios de la oficina comercial de KOTRA en Argentina señalan que el éxito de las multinacionales se debe a diversos factores externos - reactivación económica - e internos. Dentro de los últimos se destacaron la alianza con grandes superficies y cadenas de venta al público al por menor como Fravega o Grabarino, la redefinición de estrategias de marketing y publicidad (por ejemplo, mediante el auspicio de equipos de fútbol (la empresa coreana LG auspiciadora de Boca Junior) y la incorporación de mano de obra calificada argentina en cargos gerenciales, lo cual ha permitido un mejor conocimiento y manejo del mercado local. Entre los obstáculos mencionados reiteradamente por empresarios coreanos locales se destacan el tamaño del mercado argentino (sobre todo comparado con Brasil), las trabas legales, la inestabilidad política e institucional dada por el constante cambio 
de las reglas del juego, la compleja y corrupta, la estructura burocrática argentina y la baja institucionalidad de las prácticas comerciales. Debido a estos factores muchas empresas prefieran trabajar desde Brasil, que promueve la instalación de fábricas, en el Cono Sur.

En entrevista realizada en enero de 2013 al Asistente Comercial encargado de la representación de empresas de la Korean Trade Investment Promotion Agency (KOTRA) en Argentina, se destaca que en 2012 disminuyó de forma considerable el flujo de inversiones coreanas en el país como consecuencia de las políticas comerciales implementadas por el gobierno local. Frente a este cambio de escenario, el gobierno surcoreano junto con otros países han expresado ante la OMC su preocupación por las licencias de importación.

Por otro lado, se mencionó que Argentina ocupa el $58^{\circ}$ lugar en el ranking del destino de las exportaciones coreanas y el $9^{\circ}$ entre los países latinoamericanos, por lo cual las políticas de comercio exterior implementadas en Argentina no afectarían mucho el monto de las exportaciones coreanas. Asimismo, hay que recordar que la presidenta electa, Park Geun Hye, todavía no ha dado a conocer qué medidas aplicará su gobierno respecto del comercio exterior con Argentina.

\subsection{Las pequeñas y medianas empresas}

Respecto de las PYME coreanas, a pesar del éxito de compañías como
Ceragem, Medison y Arion, para que se arriesguen a invertir en el mercado local debería garantizárseles un marco legal y regulatorio estable (mínimo de 10 años). ¿Por qué invertir en Argentina en vez de Brasil que cuenta con un mercado local mucho más grande, incentivos gubernamentales y mayor estabilidad? Empresarios coreanos explican al respecto que muchas firmas coreanas prefieren instalarse en el país vecino, especialmente en la zona franca de Manaos, debido a que nuestro país no les da confianza. En este sentido, Brasil resulta mucho más tentador para los inversores: tamaño del mercado, forma parte de los BRIC, posee políticas de desarrollo coherentes y de largo plazo y zonas francas.

Los altos niveles de corrupción y la baja confianza en la estabilidad económico-política son factores claves para comprender la falta de interés en la Argentina. En el 2007, el Eximbank de Corea realizó un ranking mundial de los mejores países para realizar inversiones y la Argentina ocupó el último lugar junto a Uzbekistán. De acuerdo con el índice Doing Business del 2011 realizado por el Banco Mundial (véase el Cuadro 5), nuestro país ocupó el lugar 115, Brasil el 127, mientras que Perú en el 36 y Chile el 43. Asimismo, Argentina encabeza el índice riesgo país a nivel mundial, junto con Ecuador y Venezuela, con 535 puntos, mientras que Brasil, por ejemplo, tiene 275. Por otro lado, la nota del S\&S y Fitch para el país es B+. 
Cuadro 3: Hacer negocios en Argentina

\begin{tabular}{|c|c|c|}
\hline Temas del ranking & 2011 & 2010 \\
\hline Empezar un negocio & 142 & 137 \\
\hline Permisos de construcción & 168 & 169 \\
\hline Registro de propiedades & 118 & 114 \\
\hline Obtención de créditos & 65 & 61 \\
\hline Protección a los inversores & 109 & 108 \\
\hline Pago de impuestos & 143 & 137 \\
\hline Comercio más allá de la frontera & 115 & 113 \\
\hline Cumplimiento de contratos & 45 & 45 \\
\hline Cierre del negocio & 77 & 87 \\
\hline
\end{tabular}

Fuente: Doing Business - Banco Mundial

Los resultados que acusa este indicador concuerdan con la disconformidad expresada por los empresarios coreanos entrevistados. Las variables reiteradas son el bajo nivel de estabilidad política de las instituciones públicas, las complejidades del aparato burocrático, el elevado nivel de corrupción, la volatilidad del crecimiento, la inflación y las políticas de tipo de cambio y comerciales. Sumando la imposibilidad de negociar un TLC bilateral con el país, las trabas propias del MERCOSUR (por ejemplo en el tema automóviles) y las ventajas comparativas del país vecino, se conforma un escenario desalentador que constriñe las expectativas del inversor coreano.

Como podemos observar, las variables que definen el éxito, los temores, el fracaso o la negativa de los empresarios coreanos dependen fundamentalmente del tamaño de las compañías. Esto se debe a que la inversión que realizan en nuestro país no solo se relaciona con el riesgo que representa la inversión sino también con el del tiempo que tarda el retorno sobre la inversión realizada
(ROI). Es decir, las empresas invierten teniendo en cuenta los factores internos y externos antes mencionados, el nivel de riesgo que implica la inversión a realizarse y el tiempo de retorno de la inversión. Consecuentemente, las empresas de grandes capitales tienen mayores posibilidades de invertir, justamente porque pueden afrontar altos niveles de riesgo y porque su gran poder financiero les permite hacerles. Diferente es el caso de las pequeñas y medianas empresas, cuyo músculo financiero es mucho menor, lo que en algunos casos les impide poder hacer frente al riesgo de la inversión. Es decir, por un lado las multinacionales mantienen una política propia de inserción global y de consolidación en nuevos mercados, estas cuentan con ventajas comparativas en términos de inversión y una trayectoria más larga en lo que refiere a sus actividades en la región. Asimismo, la apelación al pragmatismo $^{12}$ les ha permitido adaptarse mejor

12 El pragmatismo ha definido el desarrollo coreano desde los años de Park Chung Hee 
a las dinámicas locales. La selección de personal local altamente calificado para ocupar puestos gerenciales y la incorporación en las sedes de Seúl de jóvenes profesionales ${ }^{13}$ originarios de los lugares en que buscan invertir, les ha permitido sortear los inconvenientes inherentes a los patrones socioculturales disímiles y adaptarse rápidamente a las técnicas de negociación, tiempos y dinámicas locales.

Estas estrategias se dan, a su vez, en un marco local muy favorable para la expansión de sus productos. Debido a que el eje de la política económica argentina actual es su orientación al

(1962-1979) cuando se consolida un modelo basado en la disociación de cuestiones de economía política de las controversias político-culturales. Esta modalidad ha caracterizado las relaciones con Japón y los Estados Unidos, como así también el comportamiento empresarial de los chaebols. Otro ejemplo claro, es la forma exitosa en que los gobiernos han logrado sobrellevar situaciones de crisis mediante una rápida adaptación a las nuevas exigencias del mercado.

13 Es interesante observar que tanto Samsung como LG (aunque más recientemente) mantienen una política activa de incorporación de jóvenes profesionales extranjeros que han realizado estudios de postgrado en Corea. Estos se integran en el grupo de trabajo relacionado con su país de origen (o, en su defecto, región más próxima) a fin de contribuir a aminorar las diferencias culturales facilitando la rápida expansión global de sus empresas. En el marco de estas políticas, LG ha lanzado un programa que consiste en el envío de los jóvenes, luego de un año de training en Corea, a la sede correspondiente en sus países de origen. consumo, LG y Samsung han encontrado un nuevo aliado en el mercado internacional. Y a pesar de las dificultades que impone el MERCOSUR en lo que respecta a la venta de vehículos, KIA Argentina logró en 2008 su record de ventas, al duplicar las del año precedente. Evidentemente es un hecho que las marcas coreanas se han posicionado positivamente en el mercado local.

Por otro lado, la experiencia de los pequeños y medianos inversionistas es mucho más contradictoria. A diferencia de las multinacionales. Estos no encuentran en el marco institucional local condiciones alentadoras que compensen los dos impedimentos estructurales clave: la distancia geográfica y el tamaño del mercado. Si embargo, hay casos de inserción exitosa en el país como el de Ceragem ${ }^{14}$, que combinando estrategias de marketing adecuadas (centradas en lo oriental) con una organización corporativa con alta capacidad de aprovechamiento de nuevos mercados, han demostrado que la clave de la expansión empresarial en el país podría estar relacionada con la creatividad para adaptarse a las exigencias locales en el marco de rubros altamente competitivos en los cuales las trabas impuestas no excedan las expectativas iniciales.

14 Empresa de camas masajeadoras que posee sedes en más de 70 países incluyendo en América Latina a República Dominicana, Costa Rica, Ecuador, Bolivia, Perú, México, Colombia, Chile, Brasil y Guatemala. En Argentina tienen sedes en la Ciudad Autónoma de Buenos Aires, Gran Buenos Aires, Tucumán, Santa Fe (capital y Rosario), Bahía Blanca y Córdoba. 
Lamentablemente, como destacan los funcionarios de KOTRA en Argentina, en 2012 las pequeñas y medianas empresas se vieron más afectadas por los cambios en las políticas de importación argentinas debido a su bajo nivel de adaptación a ellos. En la mayoría de los casos, los escasos logros registrados se han debido al éxito de la capacidad de gestión de sus importadores argentinos.

\subsection{Viejos problemas, nuevos desafios}

El comercio entre Corea y Argentina se enmarca en una clara relación NorteSur: exportación de recursos naturales por Argentina e importación de alta tecnología de Corea. Como se observa, tanto en los rubros de las empresas importadoras y exportadoras que mantienen lazos activos con el país como en el tipo histórico de flujo comercial. Por lo tanto, las firmas centradas en la venta de tecnología y electrodomésticos tienen grandes ventajas en el mercado local, que han sido aprovechadas con éxito por las multinacionales coreanas. Sin embargo, una relación comercial sostenible a largo plazo requiere una acción política coreana creativa abierta a explorar áreas de comercio intra-industrial. Asimismo, exige limar asperezas y, consecuentemente, reducir las asimetrías en las proyecciones gubernamentales. Estas han dejado huellas imborrables que apuntan a desalentar la profundización de la inversión coreana en el país. $\mathrm{Al}$ respecto, si el objetivo es realmente alentar la relación con empresas medianas consideramos fundamental trabajar en los siguientes lineamientos:

- Eficacia y agilidad en los trámites administrativos por parte del gobierno argentino.

- Transparencia de los canales institucionales locales de negociación (la corrupción revela un elevado nivel de frustración entre los empresarios coreanos que se acercan por primera vez al país.

- (Re) definición de una política comercial clara y de largo plazo (por lo menos 10 años) que proporcione estabilidad institucional y un marco legal más favorable.

- Reforzamiento de la labor de instituciones como KOTRA mediante la promoción del país en rondas de negocios.

Los desafíos actuales abarcan un amplio espectro de cuestiones relacionadas con la planificación de una estrategia adecuada a las necesidades del nuevo milenio. De acuerdo con las entrevistas efectuadas, la clave del cambio debería buscarse en controversias propias de la política local más que en diferencias de índole cultural. En este sentido, nos atrevemos a afirmar que la inhibición de las inversiones coreanas no radica en cuestiones culturales ${ }^{15}$ (ver,

15 Si bien las empresas coreanas tienen una lógica de organización sumamente jerárquica y «autoritaria» (legado confuciano) que pareciera difícil de conciliar con el ambiente local, el pragmatismo -pre- 
por ejemplo, el éxito de Corea en Medio Oriente) sino más bien en la ausencia de un marco adecuado para el desarrollo ofrecido por el gobierno local.

\section{Reflexiones finales}

Como señala Kom Won Ho, el eje de los asuntos mundiales en el siglo XXI se encuentra en Asia. El fin de la guerra fría, los cambios en el escenario político, la nueva ola de globalización e interdependencia económica permitieron que los capitales internacionales llegaran a los mercados asiáticos como inversiones productivas y de cartera provocando la baja de los precios de muchos productos debido a la competencia de Asia, el incremento posterior de la demanda de recursos naturales por parte de Asia y desequilibrios globales que han sentado las bases de la nueva dinámica comercial global. Aunque América Latina se ha convertido en un escenario muy propenso para la IED, Argentina en particular ha perdido peso económico y comercial debido al debilitamiento político, la fragmentación y el empobrecimiento social. Asimismo, y producto de políticas comerciales restrictivas y el alto nivel de inestabilidad politico-institucional, Argentina ha cedido terreno como destino de inversión extranjera frente a otros países latinoamericanos que han logrado posicionarse como destinos de interés para la inversión

viamente discutido- constituye la clave del éxito. extranjera, como Chile, Perú y Colombia. Sin embargo, y a pesar de la pérdida de relevancia de nuestro país en el escenario internacional, las relaciones con Corea se han afianzado y profundizado - tanto a nivel cultural como comercial - gracias a los factores de cambio mencionados en las secciones anteriores. Esta relación, impulsada por la estrategia coreana de inserción en América Latina y la recuperación económica argentina de los últimos años, ha logrado un éxito relativo de la política comercial, tanto de multinacionales coreanas como LG, Samsung, Hyundai o Kia como de algunas empresas medianas como Ceragen o Medison.

Lo dicho anteriormente lleva a pensar en un escenario más bien complejo porque si bien por un lado Argentina ha perdido preeminencia como destino de inversión extranjera, por el otro, salvo en 2012, la inversión de empresas coreanas en Argentina ha aumentado. Puede decirse que aunque las transformaciones del sistema económico no se han acompañado de una política comercial definida del gobierno argentino, las empresas grandes siguen viéndose favorecidas. Porque aunque institucionalmente el escenario argentino es demasiado complejo e incluso poco alentador, las empresas que han tenido la capacidad para apalancarse en un fuerte músculo financiero y adaptarse a la coyuntura institucional del país se han visto beneficiadas por el incremento del consumo interno. Por otro lado, las empresas medianas y pequeñas, a las 
que claramente les resulta más dificultoso acomodarse a los cambios institucionales en Argentina, no han podido beneficiarse de la misma manera, ya que su propia estructura financiera, por ser mucho más pequeña, no ha sido capaz de adaptarse a los cambios constantes del modelo argentino. Por esta razón, consideramos necesario que se abandone definitivamente el oportunismo coyuntural y que se plantee una acción política clara y creativa que apunte a diversificar la estructura comercial, afianzarla con nuevos mercados y a reestructurar el marco burocrático y legal interno. Solo en un nuevo escenario como este se podrán replantear y consolidar las relaciones Corea-Argentina permitiendo un flujo comercial más dinámico en el cual las empresas coreanas logren posicionarse de manera más favorable. $\mathrm{Al}$ respecto, lo que se intenta concluir es que la tendencia en materia de inversión extranjera solo podrá cambiar si Argentina ofrece un escenario político-institucional estable que permita el aumento de la IED, no solo de empresas grandes, en su mayoría multinacionales capaces de adaptarse a los cambios coyunturales, sino también de empresas medianas y pequeñas.

La transición del Atlántico al $\mathrm{Pa}$ cífico es un hecho. Es tiempo de que nuestro país adquiera conciencia y deje de lado la mera retórica, para construir una estrategia comercial con Corea que sea mucho más sólida y beneficiosa y se convierta en un escenario políticoinstitucional estable, con reglas de juego claras, que incentive la inversión extranjera, recomponiendo relaciones comerciales con socios tradicionales como lo es el país asiático.

\section{Apéndice i: Comercio CoreA-América LATINA}

Cuadro 1: Importaciones Corea-América Latina

\begin{tabular}{|c|c|c|}
\hline \multirow{2}{*}{ País } & 2010 & $2011(1 \sim 08)$ \\
\cline { 2 - 3 } & Valor & Valor \\
\hline Total & 14.644 .745 .070 & 13.767 .814 .570 \\
\hline Brasil & 4.712 .085 .317 & 4.057 .763 .324 \\
\hline Chile & 4.221 .394 .948 & 1.650 .681 .565 \\
\hline México & 1.521 .026 .525 & 1.404 .553 .620 \\
\hline Perú & 1.038 .931 .838 & 726.221 .692 \\
\hline Argentina & 794.606 .929 & 495.462 .738 \\
\hline T.Tobago & 273.303 .329 & 414.697 .808 \\
\hline Bolivia & 213.860 .738 & 309.963 .845 \\
\hline Colombia & 432.138 .641 & 236.224 .845 \\
\hline Panamá & 573.459 .883 & . \\
\hline
\end{tabular}

Fuente: Korean International Trade Association (KITA) 
M. del Pilar Alvarez y Luciana Manfredi • Una mirada crítica al sector empresarial...

Cuadro 2: Exportaciones Corea-América Latina

\begin{tabular}{|c|c|c|}
\hline \multirow{2}{*}{ País } & 2010 & $2011(1 \sim 08)$ \\
\cline { 2 - 3 } & Valor & Valor \\
\hline Total & 36.187 .293 .724 & 26.651 .102 .390 \\
\hline Brasil & 7.752 .579 .178 & 8.910 .092 .098 \\
\hline México & 8.845 .549 .442 & 6.690 .498 .002 \\
\hline Panamá & 4.053 .508 .858 & 1.963 .113 .707 \\
\hline Chile & 2.947 .053 .672 & 1.597 .392 .268 \\
\hline Colombia & 1.388 .552 .567 & 1.094 .662 .997 \\
\hline Perú & 944.438 .116 & 888.516 .984 \\
\hline Bermuda & 1.448 .204 .703 & 798.632 .090 \\
\hline Br.Virgin Is. & 1.676 .252 .534 & 725.664 .194 \\
\hline Argentina & 909.449 .459 & 698.809 .614 \\
\hline
\end{tabular}

Fuente: Korean International Trade Association (KITA)

\section{Apéndice 2: Comercio bilateral Corea-Argentina}

\begin{tabular}{|c|c|c|}
\hline$A \tilde{n} O$ & Exportaciones & Importaciones \\
\hline 1965 & 51.000 & 96.000 \\
\hline 1966 & 39.000 & 238.000 \\
\hline 1967 & No hay datos & No hay datos \\
\hline 1968 & No hay datos & 834 \\
\hline 1969 & 145.000 & 693.000 \\
\hline 1970 & 227.000 & 186.000 \\
\hline 1971 & 219.000 & 306.000 \\
\hline 1972 & 37.000 & 109.000 \\
\hline 1973 & 1.970 .000 & 346.287 \\
\hline 1974 & 6.820 .000 & 359.601 \\
\hline 1975 & 4.621 .000 & 3.037 .755 \\
\hline 1976 & 1.042 .000 & 7.064 .749 \\
\hline 1977 & 3.307 .000 & 19.681 .879 \\
\hline 1978 & 6.077 .000 & 35.728 .835 \\
\hline 1979 & 27.874 .000 & 12.869 .831 \\
\hline 1980 & 77.420 .342 & 27.988 .876 \\
\hline 1981 & 63.737 .945 & 28.444 .502 \\
\hline 1982 & 11.114 .710 & 60.137 .098 \\
\hline 1983 & 17.988 .742 & 64.300 .882 \\
\hline 1984 & 14.018 .154 & \\
\hline & & \\
\hline
\end{tabular}


Estudios Internacionales 174 (2013) • Universidad de Chile

\begin{tabular}{|c|c|c|}
\hline 1985 & 11.557 .166 & 31.507 .863 \\
\hline 1986 & 36.054 .190 & 86.744 .709 \\
\hline 1987 & 52.090 .075 & 55.514 .441 \\
\hline 1988 & 69.471 .610 & 77.864 .436 \\
\hline 1989 & 72.383 .173 & 69.733 .966 \\
\hline 1990 & 75.263 .800 & 86.290 .274 \\
\hline 1991 & 255.534 .583 & 108.178 .133 \\
\hline 1992 & 463.287 .707 & 115.707 .387 \\
\hline 1993 & 534.980 .315 & 66.568 .068 \\
\hline 1994 & 477.494 .230 & $68.267,208$ \\
\hline 1995 & 305.232 .166 & 132.364 .857 \\
\hline 1996 & 443.082 .138 & 224.343 .383 \\
\hline 1997 & 630.264 .290 & 252.843 .846 \\
\hline 1998 & 584.349 .465 & 126.175 .371 \\
\hline 1999 & 439.428 .063 & 163.380 .703 \\
\hline 2000 & 447.517 .390 & 191.313 .726 \\
\hline 2001 & 302.100 .910 & 373.870 .887 \\
\hline 2002 & 51.471 .247 & 376.419 .340 \\
\hline 2003 & 161.439 .427 & 435.669 .572 \\
\hline 2004 & 239.144 .317 & 421.498 .611 \\
\hline 2005 & 309.008 .422 & 371.754 .901 \\
\hline 2006 & 330.936 .576 & 556.020 .834 \\
\hline 2007 & 489.139 .800 & 731.532 .783 \\
\hline 2008 & 578.207 .445 & 914.771 .893 \\
\hline 2009 & 494.269 .164 & 672.256 .503 \\
\hline 2010 & 909.449 .459 & 794.606 .929 \\
\hline $2011^{1}$ & 698.809 .614 & 726.221 .692 \\
\hline
\end{tabular}

Fuente: Korean International Trade Association (KITA)

\section{Apéndice 3: Relaciones comerciales MERCOSUR-Corea}

\begin{tabular}{|c|c|c|}
\hline Año & Exporaciones & Importaciones \\
\hline 2002 & 1.354 .715 & 1.630 .600 \\
\hline 2003 & 1.370 .358 & 2.067 .506 \\
\hline 2004 & 2.101 .734 & 2.636 .365 \\
\hline 2005 & 2.828 .039 & 2.902 .738 \\
\hline 2006 & 3.516 .810 & 3.298 .554 \\
\hline 2007 & 4.112 .904 & 3.567 .769 \\
\hline 2008 & 6.697 .028 & 5.340 .989 \\
\hline
\end{tabular}


M. del Pilar Alvarez y Luciana Manfredi • Una mirada crítica al sector empresarial...

\begin{tabular}{|l|l|l|}
\hline 2009 & 5.925 .319 & 4.465 .972 \\
\hline 2010 & 8.951 .614 & 5.542 .689 \\
\hline 2011 & 9.878 .849 & 4.829 .499 \\
\hline
\end{tabular}

Fuente: Korean International Trade Association (KITA)

\section{Apéndice 4: Convenios Argentina - Corea del Sur}

\begin{tabular}{|c|c|c|c|}
\hline Firma & Vigor & Norma Aprobatoria & Acuerdo \\
\hline $\begin{array}{l}\text { Tokio, } 15 \text { de } \\
\text { febrero de } 1962\end{array}$ & $\begin{array}{l}15 \text { de febrero de } \\
1962\end{array}$ & Decreto $^{\circ} 4.542 / 64$ & $\begin{array}{l}\text { Acuerdo por notas reversales } \\
\text { estableciendo relaciones } \\
\text { diplomáticas }\end{array}$ \\
\hline $\begin{array}{l}\text { Buenos Aires, } \\
8 \text { de agosto } \\
\text { de } 1968 \\
\end{array}$ & $\begin{array}{l}4 \text { de enero } \\
\text { de } 1970\end{array}$ & 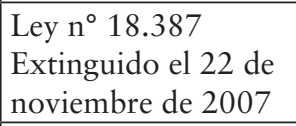 & Convenio Cultural \\
\hline $\begin{array}{l}\text { Seúl, } 14 \text { de agosto } \\
\text { de } 1972\end{array}$ & $\begin{array}{l}14 \text { de agosto } \\
\text { de } 1972\end{array}$ & & $\begin{array}{l}\text { Acuerdo por notas revérsales } \\
\text { para el otorgamiento y la } \\
\text { protección de los derechos } \\
\text { de patentes de invención, } \\
\text { modelos de utilidad, diseños } \\
\text { y marcas de fábrica a los } \\
\text { nacionales de ambos países }\end{array}$ \\
\hline $\begin{array}{l}\text { Buenos Aires, } 5 \\
\text { de mayo } 1981\end{array}$ & & & $\begin{array}{l}\text { Comunicado conjunto entre } \\
\text { el Ministro de Comercio } \\
\text { e Industria de Corea y el } \\
\text { Ministro de Comercio e } \\
\text { Intereses Marítimos de la } \\
\text { República Argentina }\end{array}$ \\
\hline $\begin{array}{l}\text { Buenos Aires, } \\
28 \text { de octubre } \\
\text { de } 1991\end{array}$ & $\begin{array}{l}28 \text { de octubre } \\
\text { de } 1991\end{array}$ & & $\begin{array}{l}\text { Memorándum de } \\
\text { Entendimiento para el } \\
\text { Establecimiento de una } \\
\text { Comisión Mixta }\end{array}$ \\
\hline $\begin{array}{l}\text { Buenos Aires, } \\
16 \text { de junio } \\
\text { de } 1992 \\
\end{array}$ & $\begin{array}{l}16 \text { de julio } \\
\text { de } 1992\end{array}$ & & $\begin{array}{l}\text { Acuerdo por canje de notas } \\
\text { sobre visado múltiple para } \\
\text { hombres de negocios }\end{array}$ \\
\hline $\begin{array}{l}\text { Seúl, } 17 \text { de mayo } \\
1994\end{array}$ & $\begin{array}{l}24 \text { de septiembre } \\
\text { de } 1996\end{array}$ & Ley $n^{\circ} 24.682$ & $\begin{array}{l}\text { Acuerdo para la promoción y } \\
\text { la protección recíproca de las } \\
\text { inversiones }\end{array}$ \\
\hline $\begin{array}{l}\text { Buenos Aires, } \\
30 \text { de agosto de } \\
1995\end{array}$ & $\begin{array}{l}9 \text { de noviembre } \\
\text { de } 2000\end{array}$ & Ley 25.303 & Tratado de Extradición \\
\hline $\begin{array}{l}\text { Seúl, } 29 \text { de } \\
\text { septiembre de } \\
1995\end{array}$ & & & $\begin{array}{l}\text { Memorándum de } \\
\text { Entendimiento sobre } \\
\text { Consultas Periódicas de Alto } \\
\text { Nivel }\end{array}$ \\
\hline
\end{tabular}


Estudios Internacionales 174 (2013) • Universidad de Chile

\begin{tabular}{|c|c|c|c|}
\hline $\begin{array}{l}\text { Buenos Aires, } 9 \\
\text { de septiembre de } \\
1996\end{array}$ & $\begin{array}{l}19 \text { de septiembre } \\
\text { de } 1997\end{array}$ & Ley $n^{\circ} 24.860$ & $\begin{array}{l}\text { Acuerdo sobre Cooperación } \\
\text { en los Usos Pacíficos de la } \\
\text { Energía Nuclear }\end{array}$ \\
\hline $\begin{array}{l}\text { Buenos Aires, } 9 \\
\text { de septiembre de } \\
1996\end{array}$ & $\begin{array}{l}15 \text { de enero de } \\
2004\end{array}$ & Ley 25.834 & $\begin{array}{l}\text { Acuerdo sobre Servicios } \\
\text { Aéreos }\end{array}$ \\
\hline $\begin{array}{l}\text { Seúl, } 31 \text { de } \\
\text { octubre de } 2000\end{array}$ & $\begin{array}{l}12 \text { de febrero de } \\
2003\end{array}$ & Ley 25.702 & $\begin{array}{l}\text { Acuerdo de cooperación } \\
\text { científica y Tecnológica }\end{array}$ \\
\hline $\begin{array}{l}\text { Buenos Aires, } 1 \\
\text { de Septiembre de } \\
2003\end{array}$ & $\begin{array}{l}1 \text { de septiembre } \\
\text { de } 2003\end{array}$ & & $\begin{array}{l}\text { Acuerdo sobre Cooperación } \\
\text { Bilateral en Materia Pesquera }\end{array}$ \\
\hline $\begin{array}{l}\text { Buenos Aires, } 1 \\
\text { de abril de } 2004\end{array}$ & $\begin{array}{l}31 \text { de julio de } \\
2004\end{array}$ & & $\begin{array}{l}\text { Acuerdo por canje de } \\
\text { notas para la supresión de } \\
\text { visado en los pasaportes } \\
\text { diplomáticos yoficiales. }\end{array}$ \\
\hline $\begin{array}{l}\text { Buenos Aires, } 15 \\
\text { de noviembre de } \\
2004\end{array}$ & $\begin{array}{l}25 \text { de octubre de } \\
2006\end{array}$ & $\begin{array}{l}\text { Acuerdo de } \\
\text { cooperación } \\
\text { económica y } \\
\text { comercial } \\
\end{array}$ & \\
\hline $\begin{array}{l}\text { Buenos Aires, } 15 \\
\text { de noviembre de } \\
2004\end{array}$ & $\begin{array}{l}15 \text { de noviembre } \\
\text { de } 2004\end{array}$ & & $\begin{array}{l}\text { Acuerdo sobre cooperación } \\
\text { en los campos de recursos de } \\
\text { energía y minería }\end{array}$ \\
\hline $\begin{array}{l}\text { Buenos Aires, } 15 \\
\text { de noviembre de } \\
2004\end{array}$ & $\begin{array}{l}15 \text { de noviembre } \\
\text { de } 2004\end{array}$ & $\begin{array}{l}\text { Extinguido el } 15 \text { de } \\
\text { noviembre de } 2009\end{array}$ & $\begin{array}{l}\text { Acuerdo sobre cooperación } \\
\text { en el área de tecnología } \\
\text { de la información y las } \\
\text { comunicaciones }\end{array}$ \\
\hline $\begin{array}{l}\text { Buenos Aires, } 15 \\
\text { de noviembre de } \\
2004\end{array}$ & $\begin{array}{l}22 \text { de noviembre } \\
\text { de } 2007\end{array}$ & 26.284 & $\begin{array}{l}\text { Convenio de cooperación } \\
\text { cultural y educativa }\end{array}$ \\
\hline $\begin{array}{l}\text { Buenos Aires, } 15 \\
\text { de noviembre de } \\
2004\end{array}$ & $\begin{array}{l}15 \text { de noviembre } \\
\text { de } 2004\end{array}$ & $\begin{array}{l}\text { Extinguido } 15 \text { de } \\
\text { noviembre de } 2009\end{array}$ & $\begin{array}{l}\text { Memorando de } \\
\text { entendimiento para la } \\
\text { cooperación agrícola, } \\
\text { científica y técnica } \\
\end{array}$ \\
\hline $\begin{array}{l}\text { Seúl, } 31 \text { de agosto } \\
\text { de } 2009\end{array}$ & & $\begin{array}{l}\text { Mensaje no } 93 \text { del } \\
1 \text {-II-2011 }\end{array}$ & $\begin{array}{l}\text { Tratado sobre asistencia legal } \\
\text { mutua en materia penal }\end{array}$ \\
\hline $\begin{array}{l}\text { Buenos Aires, } 5 \\
\text { de noviembre de } \\
2010\end{array}$ & $\begin{array}{l}5 \text { de noviembre } \\
\text { de } 2010\end{array}$ & & $\begin{array}{l}\text { Acuerdo por notas verbales } \\
\text { para corregir el texto en } \\
\text { coreano del «Tratado sobre } \\
\text { asistencia mutua en materia } \\
\text { penal del } 31 \text { de agosto de } \\
2009 »\end{array}$ \\
\hline $\begin{array}{l}\text { Buenos Aires, } 6 \\
\text { de diciembre de } \\
2010\end{array}$ & & & Declaración conjunta \\
\hline
\end{tabular}

Fuente: Ministerio de Relaciones Exteriores de la República Argentina 


\section{BIBLIOGRAFÍA}

Barkema, H., y J. Bell. (1996). Foreign entry, cultural barriers, and learning. Strategic Management Journal, Vol. 17, 151-166.

Choi, D. (1998), Hacia nuevas relaciones económicas entre la República de Corea y América Latina en Moneta, Carlos Juan y Gerardo Orlando Noto (eds.) «Dragones, Tigres y Jaguares: Relaciones América Latina/Asia-Pacífico más allá de la crisis», Buenos Aires: Corregidor, pp. 151-183.

Girado, G. (2003), Comercio Argentina/Asia Pacífico: una carrera de obstáculos, Buenos Aires, Corregidor.

Kim, W. (2000), East Asian-Latin American Economic Relations: A Korean Perspective after the International Financial Crisis, en KIEP Working Paper 00-04.

Kim, W. (2008), América Latina en el siglo XXI: reflexiones criticas desde Asia del Este, Nueva Sociedad, 214, pp. 36-46

Kim, W. (2009), Estado versus Mercado en América Latina, Nueva Sociedad, 221, pp. 151-165.

Lin, H. (2000). Choice of market entry mode in Emerging markets: influences on entry strategy in China, Journal of Global Marketing, Vol.14.

Mera, C. (1998), La inmigración coreana en Buenos Aires. Multiculturalismo en el espacio urbano, Buenos Aires, EUDEBA.

Mera, C. (2007), Panorama general y reflexiones acerca de las relaciones entre Argentina y Corea, en Revista de Estudios Internacionales, 156 pp. 67-82.

Parmigiani, A. (2007). Why do firms both make and buy? An investigation of concurrent sourcing. Strategic Management Journal, 28(1): 285-311.

Root, F. (1998) Entry Strategies for International Markets, Nueva York, Jossey-Bass.

Yoo, J. (1999), Real Success, Financial Fall: A Reassessment of the Korean Dynamism, Seúl, Ewha Woman's University Press.

\section{Artículos en Internet:}

Chacón, Alejandra (2008), Perceptions of the South Korean foreign policy towards

Argentina, Brazil and Chile, http://www. international.ucla.edu/media/files/SRGChacon.pdf

Martini, Maria (2008), Negociaciones internacionales. Reflexiones sobre Corea, Edición impresa COMERCIO EXTERIOR - LA NACION, http://www.cei.gov.ar/pdf/ prensa/nacion40508.pdf

Kim, Won Ho (2000), La globalización de la economía coreana y América Latina, http:// www.asiayargentina.com/cari-12.htm

Kim, Christine (2010), Korea seeks out strong relations with Argentina, Korea Joongang Daily, http://joongangdaily.joins.com/ article/view.asp?aid $=2927027$

Mera, Carolina (2010), La inmigración coreana en Buenos Aires. Historia y actualidad, Ponencia presentada en el XI Congreso Nacional de aladaA, Universidad de Colima, México, http://ceaa.colmex.mx/ aladaa/imagesmemoria/carolinamera.pdf

Paz, Gonzalo, (2000), Las relaciones entre Corea y la Argentina. Desarrollo y perspectivas, Cuadernos de Estudio de las Relaciones Internacionales Asia-PacíficoArgentina, N1, pp.145-160, http://www. cari.org.ar/pdf/asia-pacifico.pdf.

\section{Datos estadísticos en Internet:}

Doing Business: (http://www.doingbusiness. org), consultado el 27 de agosto de 2012

Korean Trade Internacional Association (KITA): Korean Trade Internacional Association (http://www.kita.org), consultado el 28 de agosto de 2012

Ministerio de Comercio y Relaciones Exteriores de Corea del Sur: Ministerio de Comercio y Relaciones Exteriores de Corea del Sur (http://www.mofat.go.kr/english/ regions/latinamerica/20070803/1_328. jsp), consultado el 29 de agosto de 2012.

Oficina Comercial de la Embajada de Corea del Sur en Argentina (KOTRA): Korea 
Business Center Buenos Aires (http:// kotra.org.ar/index.jsp), consultado el 27 de agosto de 2012.

Ministerio de Relaciones Exteriores de la República Argentina: Biblioteca Virtual (http://tratados.cancilleria.gob.ar/busqueda.php? consulta $=$ si\&modo $=c)$, consultado el 28 de agosto de 2012. 